\title{
Chabines et métisses dans l'univers antillais
}

Entre assignations et négociations identitaires

Stéphanie Mulot

\section{(2) OpenEdition}

Journals

Édition électronique

URL : https://journals.openedition.org/clio/7447

DOI : $10.4000 /$ clio. 7447

ISSN : 1777-5299

Éditeur

Belin

Édition imprimée

Date de publication : 1 janvier 2008

Pagination : 115-134

ISBN : 978-2-85816-973-3

ISSN : 1252-7017

Référence électronique

Stéphanie Mulot, "Chabines et métisses dans l'univers antillais », Clio. Histoire, femmes et sociétés [En ligne], 27 | 2008, mis en ligne le 05 juin 2010, consulté le 08 décembre 2022. URL : http:// journals.openedition.org/clio/7447 ; DOI : https://doi.org/10.4000/clio.7447

Ce document a été généré automatiquement le 29 septembre 2020.

Tous droits réservés 


\title{
Chabines et métisses dans l'univers antillais
}

Entre assignations et négociations identitaires

\author{
Stéphanie Mulot
}

1 Quelles sont la perception et la signification du métissage aux Antilles françaises ? Si le métissage et la créolisation sont au fondement des dynamiques sociales et culturelles de ces sociétés nées d'une rencontre violente entre l'Europe, l'Afrique et l'Amérique, une analyse circonstanciée permet de montrer que toutes les formes de métissage n'ont pas la même reconnaissance sociale, ni la même signification symbolique. La violence fondatrice de l'esclavage et les rapports de domination (de race, de classe et de genre) qui lui ont survécu modifient le regard porté par la société sur les différents types de métissage.

2 J'aimerais ainsi explorer deux figures antillaises particulières: les «chabins » et les «métis ». En analysant l'imaginaire dans lequel se nourrissent de telles catégorisations, il s'agira de montrer, d'une part, comment ces étiquetages produisent des assignations identitaires bien au-delà de la seule désignation de la couleur et comment, d'autre part, ils modifient la nature des rapports sociaux établis avec les personnes ainsi désignées. Enfin, en analysant les interactions qui se tissent sur la base de tels étiquetages, je voudrais mettre en évidence que derrière ces assignations premières, se met en œuvre une véritable négociation identitaire qui repose sur la perméabilité des catégories raciales et sur la capacité des individus à en jouer.

\section{Les chabins : la construction sociale d'un métissage valorisé}

\section{Les traces du préjugé de couleur}

3 Comme plusieurs chercheurs ont pu le montrer ${ }^{1}$, la société antillaise s'est organisée pendant plusieurs siècles selon une échelle socio-raciale instituée par la colonisation européenne, qui a perduré après l'abolition de l'esclavage, et qui s'est notamment traduite par une hiérarchisation et une classification très pointues des différents types 
d'individus nés de la rencontre des Blancs, des Noirs et de leurs descendants. Le métissage est vite apparu dans la société coloniale comme un moyen de promotion sociale pour des individus qui pouvaient ainsi espérer que leurs enfants échappassent à l'enfer de l'esclavage. Ces types de mélange ont été définis en fonction de leur apparence physique (le phénotype), ainsi que de leurs supposés "natures", tempéraments et capacités d'adaptation et d'assimilation, critères qui fondaient pendant la période coloniale les attributs et caractéristiques de la race ${ }^{2}$. Une terminologie issue du vocabulaire zoologique a été construite pour désigner les mulâtres, les mameloucs, les quarterons, les câpres, les griffes, les sacatras... et les distinguer selon leur degré de miscégénation ${ }^{3}$.

4 Les termes désignant les types raciaux ont progressivement évolué pour désigner des classes sociales puis des pratiques sociales. Ainsi, le terme " mulâtre », initialement utilisé pour les individus nés d'une mère noire et d'un père blanc, est devenu au XIX ${ }^{e}$ siècle un étiquetage qualifiant l'ensemble des libres de couleur soucieux de leur distinction sociale ${ }^{4}$ , à travers des comportements sociaux particuliers (parler français, usage de la citoyenneté, inscription dans la légitimité, activité professionnelle notable...). Par ailleurs, la diffusion du métissage dans la société antillaise au fil des siècles a rendu caduques des terminologies pointues qui avaient pour objectif de distinguer et séparer les catégories du métissage selon le préjugé et la ligne de couleur. La tendance contemporaine serait plus celle d'un nivellement terminologique. Pourtant chaque terme porte toujours une symbolique particulière.

5 Parmi tous les types ainsi édifiés par l'arbitraire du visuel, bien que n'ayant été repris que très tard dans les textes des voyageurs, celui des «chabins » et des " chabines $\|^{5}$ occupe une place fascinante et emblématique dans la société et l'imaginaire antillais, dont ils sont la fierté. Sans jamais adhérer aux classifications raciales, je me propose de présenter ici les catégories existant encore dans les représentations, les discours et les pratiques populaires au début du XXI ${ }^{e}$ siècle en Guadeloupe, qui pourraient parfois sembler tout droit héritées des descriptions anatomiques et anthropologiques du XIX ${ }^{e}$ siècle.

\section{Une apparence et un caractère remarquables : l'alliance des races}

6 L'identification des chabins s'appuie sur leur apparence pour le moins spectaculaire dans l'univers noir et métissé des Antilles. Cette apparence repose sur l'alliance entre une morphologie et des traits négroïdes au niveau du visage (le cheveu crépu, les lèvres épaisses, le nez épaté, le visage rond), mais aussi du corps (cambrure des reins, largeur des hanches, rebondi des fesses, arrondi du buste ${ }^{6}$, épaisseur des lèvres sexuelles), et une pigmentation claire (les cheveux et les poils blonds, les yeux gris, verts ou noisette, une couleur de peau claire et parfois laiteuse). Cette conjonction de traits, que le discours sur les races avait opposés, s'affiche ainsi dans ce que Michel Leiris avait décrit comme étant: « au lieu d'un amalgame, une combinaison paradoxale de traits des races noire et blanche $^{7}$ ». Dans l'univers contemporain, ce mélange est considéré par la population avec bonheur, amusement et fierté, car il est perçu comme conciliant avec beauté, succès et équité des caractéristiques blanches et noires, sans que l'une ne s'impose plus que l'autre.

7 Mais la définition sociale des chabins ne se limite pas à leur phénotype. Leur caractère et leur tempérament, notions également issues des théories de la race $\mathrm{du} \mathrm{XvI}^{\mathrm{e}}$ siècle, sont décrits comme étant bien différents de ceux des Nègres ou des Blancs. Le tempérament des chabins est dit sulfureux. Ils sont perçus comme des individus au caractère colérique, explosif voire volcanique, enflammé, prêts à sortir de leurs gonds à la première étincelle. 
Selon Raphaël Confiant ${ }^{8}$, qui s'auto-identifie au chabin, ils se voient attribuer les caractéristiques que l'on réservait aux roux en France: imprévisibilité, irritabilité, susceptibilité, mauvais caractère. La littérature leur confie d'ailleurs souvent des rôles de personnages au caractère trempé voire inquiétant ${ }^{9}$. Parfois «devineuses", " quimboiseuses » (sorcières), ou séductrices, envoutantes et fatales, les chabines quant à elles dégagent une prestance qui impressionne.

Par ailleurs, cette édification du caractère supposé des chabins repose sur l'attribution d'un prétendu tempérament «naturel »: leur « nature » serait d'être « cho » (« chauds ») dans une typologie qui oppose sur cette échelle de la chaleur au sommet les Noirs et à la base les Blancs. C'est pourquoi leur sang chaud, circulant sous une peau claire, ne pourrait supporter trop longtemps le soleil, ni les plats épicés. Cette chaleur se manifesterait physiquement par des rougissements spectaculaires au niveau du visage, état que peu de personnes noires connaissent, et par des accès de colères et d'excitation. Si le caractère trempé des chabins est souligné, le plus souvent il est aussi rapporté avec amusement: en effet, ce personnage fascine et trouble, mais il amuse plus qu'il ne dérange, il fait rire et sourire plus qu'il n'inquiète. On le titille même à souhait, pour voir s'exprimer cette caractéristique attendue, confirmant la «nature » commune à tous les chabins.

Ici aussi, une codification très pointilleuse distingue différents types de chabins et particulièrement de chabines: de la chabine "takté kon dend» (tachetée comme une dinde, avec des taches de rousseur et des grains de peau ${ }^{10}$ à la chabine «kalasasa ${ }^{11}$ en passant par la chabine "pwel si » (aux poils durs) ${ }^{12}$. Et, chabine parmi les chabines, la "chabine dorée ", qui jouit d'un prestige énorme et d'un pouvoir de séduction hors du commun. Elle se reconnaît à sa luminosité, due à ses cheveux blonds dits «jaunes » ou " chivé soley » (cheveux soleil), « cheveux d'or ", à sa pilosité toute blonde ou rousse ${ }^{13}$, à ses yeux gris-vert, dans un corps considéré comme étant celui d'une «négresse » du fait de ses traits négroïdes, et surtout à un tempérament caractériel et sexuel très chaleureux voire explosif.

\section{Un tempérament sexuel singulier : l'alliance des genres}

Dans cette entreprise de naturalisation du social, cette même « chaleur naturelle » qui est censée caractériser les chabins serait la cause d'un tempérament sexuel actif et dynamique chez les chabines. En effet, les femmes chabines sont réputées pour des aptitudes sexuelles qui les distingueraient des autres types de femmes et des chabins. Leur réputation de symboles sexuels repose sur le fait qu'elles seraient des femmes qui " font ça mieux » que les autres, qui «bougent mieux », qui « sont plus énergiques et plus douces en même temps ", et qui recèlent de nombreuses habiletés pour exciter un homme : « chabin ka modé zorey " ( les chabines mordent les oreilles»), elles sont donc entreprenantes, « lanmou a yo pli dous » (« leur façon de faire l'amour est plus douce »), elles manifestent de la tendresse. Elles auraient ainsi un savoir faire naturel, du fait de leur type : «Yo ni ras » (« elles ont de la race »), faisant d'elles des femmes beaucoup plus jouissives que les autres. Ce seraient des femmes capables de mener un homme sexuellement « sé yo ki ka kontrol » (« c'est elles qui contrôlent »), et plaisent justement par ce dynamisme qui leur est autorisé et reconnu. Par ailleurs, le corps des chabines serait le territoire d'une volupté sans égale. Comme les Indiennes, elles sont même parfois considérées comme des «femmes-fontaines » capables de produire une lubrification et 
une jouissance vaginales que d'autres femmes ne connaîtraient pas ${ }^{14}$. Pour tout cela, elles manifesteraient plus leur plaisir que les autres femmes et surtout seraient capables d'en donner davantage. Elles se distinguent aussi des chabins, connus essentiellement pour leur séduction phénotypique, mais qui ne sont pas censés détrôner les Noirs dans leurs légendaires talents sexuels.

Cette attribution d'un rôle sexuel actif et chaleureux tranche complètement avec les règles de construction des identités sexuelles très hétéro-normées, où la respectabilité des femmes se construit, en droits plus qu'en faits, sur leur discrétion sexuelle, leur inhibition, leur soumission et leur fidélité, par opposition à la réputation des hommes qui doit leur permettre d'afficher ostensiblement leurs capacités sexuelles. En outre, si les représentations populaires reconnaissent aux femmes noires une "nature " chaude, l'éducation des jeunes filles a pour dessein de contenir cette chaleur le plus possible, pour leur permettre de trouver un compagnon qui voudra bien les épouser. Or, dans une société matrifocale où les unions sont consensuelles et la nuptialité faible, le mariage apparait cependant comme un critère de reconnaissance sociale extrêmement précieux, puisque rare. Ainsi, ce souci de discrétion est imposé aux jeunes femmes dont les mères espèrent que les conduites ne viendront pas nourrir les commérages publics, même si la transgression des interdits est de rigueur.

Les termes les plus insultants sont adressés aux femmes qui se risqueraient sur le terrain d'une sexualité active et assumée et qui ne respecteraient pas les frontières du genre: "ochan, malprop, rat, cochoni, visièz...», (pressée, malpropre, ratte, cochonnerie, vicieuse) sont autant de termes que résume le qualificatif «salop» (salope) et que les hommes adressent facilement aux femmes qu'ils veulent ainsi mépriser. Cette construction sociale du genre est censée amener les femmes qui veulent briller par leur respectabilité à rester prudes et timides devant les choses du sexe et à ne pas faire preuve de trop d'aisance ni connaissance en ce qui concerne la sexualité. Ce qui n'est pas sans poser problèmes ni paradoxes dans cette société où la concurrence féminine discrimine aussi les femmes selon leurs capacités à garder un partenaire auprès d'elles, notamment sexuellement. C'est alors plutôt dans leur rôle de maîtresse que dans leur rôle d'épouse que les femmes laissent exprimer une liberté sexuelle que la légitimité et la respectabilité de leur mariage interdisent, et que leurs époux ne sauraient exiger ni même parfois tolérer.

Ce souci de discrétion s'oppose complètement à la norme de réputation et d'exhibition virile à laquelle sont soumis les garçons de leur côté, qui leur permet de vanter leurs conquêtes supposées ou réelles et de se faire valoir dans l'arène de la compétition sexuelle en multipliant les partenaires. Dans la construction des identités sexuées, le souci permanent de montrer ostensiblement sa virilité, qui témoigne de la fragilité des fondements de l'identité masculine, rime avec une homophobie généralisée. Le spectre de l'homosexuel, " makomè » ${ }^{15}$ moqué et ridiculisé, est brandi dans l'éducation des garçons par les femmes et par les mères, comme un contre-modèle méprisable, comme limite à ne pas franchir ${ }^{16}$.

La sexualité chaleureuse qui est reconnue à la chabine lui confère donc une place privilégiée dans les rapports sociaux de sexes, en lui permettant de se positionner sur un registre masculinisé de la sexualité : celui de l'activité et de la prise d'initiative. Le personnage de la chabine ainsi construit socialement brouille quelque peu les distinctions de genre sur lesquelles repose l'éducation des enfants (figure 1). Ce brouillage est perçu comme excitant et réjouissant par les hommes qui se positionnent d'emblée dans des 
rapports de séduction avec ces femmes à l'apparence prometteuse, qui font figure de proies pour les uns et de prédatrices pour leurs rivales.

\section{La chabine au cœur de la racialisation des sexes}

Les recherches des historiens et philosophes ont montré comment le sexe et la race ont été confondus dans les descriptions des femmes et hommes de couleur au fil des siècles ${ }^{17}$. Là encore, une échelle des caractéristiques sexuelles attribuées aux Noires, aux Blanches et femmes de couleur s'est construite en considérant la chaleur et le tempérament des types en question. La description de l'anatomie des sexes, de leur physiologie et de leurs compétences, selon les différents types raciaux, figure au cœur du regard naturaliste et anthropologique de l'époque coloniale, tant la question de la sexualité et de la reproduction y est centrale.

Une certaine fascination naît et demeure pour la sexualité de la femme de couleur créole, principalement pour la mulâtresse, séductrice née, par opposition à la créole blanche, respectable, confinée dans une sexualité légitime et incorruptible, et à la négresse africaine présentée sous les traits d'une sauvage ébouriffée et innocente.

Aux femmes blanches, épouses et mères légitimes, sont associés tous les éléments de la respectabilité, alors que sont réservés aux femmes de couleur les tropes de la sexualité débridée et de la perversité, en quoi elles s'opposent à la femme africaine, présentée sous les traits d'une innocence sauvage $(. . .)^{18}$.

Dans cette classification, une vision très sexualisée est donnée de la mulâtresse : «Dans tous les temps (...), les mulâtresses ont été les prêtresses de l'amour illégitime et ont formé dans l'empire des femmes une secte redoutable $»^{19}$. Elle semblait donc occuper un entre-deux, celui de l'érotisme encouragé, dont la chabine aurait peut-être hérité (figure 2).

18 Si cette échelle de valeurs s'est aujourd'hui inversée, sous les effets de migrations et d'un tourisme qui ont permis de rendre les femmes blanches plus accessibles et, par le fait, moins respectables que ne l'avaient été les femmes des Blancs durant la période coloniale, l'opposition racialisée des comportements sexuels semble perdurer (figure 3). Au cœur de cette opposition de race et de genre, les chabines occupent une place bien convoitée, mais néanmoins très surveillée. Elles sont condamnées à offrir la sexualité et le caractère que l'on attend d'elles.

Fig. $\mathrm{n}^{\circ} 1$ : La représentation des rôles sexuels dans l'éducation

Fig. $\mathrm{n}^{\circ} 2$ : L'échelle de valeur des comportements sexuels féminins (époque coloniale

Fig. $n^{\circ} 3$ : L'échelle de valeur des comportements sexuels féminins (époque contemporaine)

Cet attrait privilégié dont bénéficient les chabines les place en même temps au cœur des rivalités féminines. Elles sont perçues comme capables de donner plus de plaisir à un homme, tout en restant respectables, mais surtout comme capables de prendre leur plaisir et de ne pas s'en cacher ! Cette exception représente un idéal fantasmatique pour les hommes puisqu'il leur permet non seulement de satisfaire leur volonté de promotion sociale par la couleur de leur partenaire, sans avoir recours à une femme blanche aujourd'hui symbole de "malpropreté », mais en restant avec une véritable Antillaise, et 
sans avoir non plus à subir le manque de dynamisme sexuel supposé des autres Antillaises.

\section{L'injonction sociale à se conformer au rôle de chabin}

20 Ainsi, cette négresse paradoxale qu'est la chabine est socialement construite comme une véritable alliance non seulement au niveau du phénotype, du «tempérament » et de la nature (critères qui ont servi à déterminer celui de race), mais surtout au niveau du genre. Plus que le chabin, la chabine fascine car elle fait exception dans l'univers souvent hiérarchisé des attributs raciaux et sexuels. Si les chabins sont connus pour avoir des tempéraments explosifs et les chabines pour être des femmes plus jouissives que d'autres, c'est véritablement parce que leur éducation et le regard social leur en laissent le droit, quand ils sanctionnent l'attitude d'autres personnes qui transgresseraient les normes sociales. En effet, outre le fait qu'ils font la fierté de leur famille, les enfants chabins bénéficient d'une attention et d'une indulgence peu courantes dans un contexte d'éducation souvent très sévère. Le fait qu'un enfant soit considéré comme chabin peut l'immuniser face aux corrections subies par d'autres, au motif que sa "chabinité » peut être perçue comme étant la cause de son attitude, et qu'elle doit être encouragée plus que réprimée : «Ah! Tu es bien une chabine ! » lui adresse-t-on fièrement et gentiment.

Outre cette tolérance, les chabins sont l'objet d'une réelle incitation à prouver qu'ils sont bien ce que l'on attend d'eux. Non seulement les parents, mais aussi tout l'entourage ne manqueront pas de rappeler à ces enfants ce qu'ils sont, et donc ce qu'ils se doivent d'être, ne serait-ce qu'en les appelant non par leur prénom mais par le sobriquet «chabin » ou " chabine " voire " bibine ${ }^{20}$. De même, toute réactivité ou opposition de leur part, ou toute contradiction quant à leur nature supposée, peut être rapidement interprétée comme la preuve évidente de leur nature "chaude » et explosive, sans que leur désaccord puisse être perçu comme fondé sur autre chose que leur supposée identité naturelle. Cette essentialisation de l'apparence se transforme donc en une véritable injonction identitaire, dans laquelle chabins et chabines sont condamnés à être ce que le discours prétend qu'ils sont.

Cette injonction identitaire peut se traduire par des choix éducatifs faits pour garantir la conformité de l'enfant à son statut de chabin, qui lui confère une place sociale favorisée dans la hiérarchie socio-raciale antillaise, du fait de l'ouverture avec laquelle il sera reçu dans les différentes sphères de la vie publique. La clarté de peau, la " po chapé » (peau sauvée), a longtemps été un gage de réussite sociale aux Antilles et a fait disparaître nombre d'obstacles rencontrés par les Noirs notamment dans la carrière professionnelle et matrimoniale. Dans une société où l'apparence et la couleur opèrent comme un véritable pedigree ${ }^{21}$, la chabinité est un passeport. Laura a pu le constater à son profit : née d'une mère négresse et d'un père mulâtre qui n'ont jamais cohabité, cette chabine a été sortie de son environnement maternel défavorisé pour être élevée par sa marraine, sa tante paternelle, et lui conférer une éducation plus digne de son rang.

Des personnages politiques antillais ont eu, eux aussi, à subir ou profiter de cette assignation identitaire dans leurs activités. Lucette Michaux-Chevry, ex-ministre, exdéputée maire de Basse-Terre et ex-présidente du conseil régional de Guadeloupe, ainsi que Alfred Marie-Jeanne, président du conseil régional de Martinique, ont probablement vu leur carrière et leur audience modifiées par le fait qu'ils sont perçus avant tout comme des chabins. Une tolérance face à leurs coups de colère, une admiration pour leurs 
poignes de fer et leurs excès, une fierté face à leur résistance, tout comme un amusement moqueur face à leurs déboires ont nourri les croquis des caricaturistes, les commentaires des journalistes et les éloges de la population.

\section{De l'injonction à la négociation identitaire}

\section{Les chabins sont-ils des métis ? : une origine blanche imprécise et oblitérée}

Contrairement à d'autres catégories de métissages où la prédominance de l'une des origines est manifeste dans l'apparence visuelle, les chabins s'imposent comme les fruits d'une rencontre équitable et pacifique entre deux catégories raciales - Noir et Blanc - et leurs caractéristiques, que tout oppose et hiérarchise par ailleurs. Rencontre qui se distingue par l'étrange et spectaculaire harmonie du résultat, dans ce qui pourrait sembler, a priori, une apologie du métissage. Toutefois, il faut noter que la fascination provoquée par ces personnages tient au fait que les chabins étaient traditionnellement considérés comme étant les rejetons de deux Antillais de couleur, contrairement aux individus qualifiés aujourd'hui de «métis » par exemple, ayant un parent blanc et l'autre noir ${ }^{22}$. Dans ces représentations d'une «chabinité " proprement antillaise, distinctes de celles du métissage direct, l'origine blanche, symbole de domination et de dépossession, tout autant que de pouvoir et de reconnaissance, effrayante bien que séduisante, et l'origine nègre, symbole d'infériorisation et d'humiliation, tout autant que de résistance et d'honneur, valeureuse bien que dévalorisée, prises toutes deux dans un véritable oxymoron identitaire de la fierté honteuse, sont dissoutes dans les dédales d'une généalogie dont on ne saurait décrire les méandres avec précision. L'ancêtre responsable de l'apport blanc n'est pas nécessairement mis en avant, ni identifié de façon précise, parfois il est même oublié, effacé, alors que les généalogies font pourtant par ailleurs l'objet d'un souci pointilleux.

25 Ainsi, la non-identification des apports originels premiers dans l'histoire des chabins conforte l'idée d'un dépassement nécessaire du métissage et de ses ancrages, au profit d'une nouvelle dimension identitaire élaborée sur la base d'un métissage déjà sédimenté de longue date, d'une créolisation aux manifestations imprévisibles ${ }^{23}$. La dimension conflictuelle du métissage, liée aux oppositions tacites entre des catégories raciales qui s'attirent néanmoins, semble trouver une forme de résolution dans la reconnaissance valeureuse de la chabinité, où les apports initiaux sont pacifiquement assimilés, sans qu'il soit possible de les identifier ni, par conséquent, de les dissocier.

Cependant, il faut nuancer. Les chabins, contrairement aux métis, procurent aussi une fierté incommensurable à leurs parents et à la société tout entière parce qu'ils offrent aux Noirs la possibilité de produire du clair sans passer directement par le Blanc, et sans se risquer à quelque compromission, tout en gardant des attributs noirs valorisants. Pour comprendre l'importance de cette alchimie, il faut prendre en compte la symbolique et les représentations de l'origine du métissage aux Antilles.

\section{Le métissage désiré contre le viol fondateur}

J'ai eu l'occasion de montrer ${ }^{24}$ que deux versions sont présentes dans la littérature et dans l'imaginaire antillais pour figurer les fondements du métissage. La première évoque une 
rencontre faite de désir entre une esclave noire et un homme blanc. C'est une version similaire qui est aussi présente dans d'autres sociétés colonisées, notamment dans le récit mythique des amours de Pocahontas Matoaka (1597-1617), première indigène à ouvrir la voie du métissage chez les Indiens d'Amérique. La seconde, celle d'un viol fondateur, met en scène une histoire beaucoup plus violente et traumatisante puisque l'esclave africaine déportée vers l'Amérique est violée, quotidiennement et systématiquement, par les marins blancs sur les négriers. Ces deux versions ne prennent pas sens de la même façon pour les Antillais contemporains et entraînent des modalités de construction identitaire différentes.

Paradoxalement, ce qui est en jeu dans ces deux versions, c'est non seulement la destinée de la femme noire, mais aussi la place de l'homme noir, qui fait pourtant figure d'absent. Dans la première version, il est réduit à un homme dépossédé, impuissant et castré, par l'esclavage qui l'assujettit, par le maitre qui séduit sa congénère et par la femme qui le trahit en lui préférant le charme physique et le statut social de l'homme blanc. Dans le mythe du viol fondateur, l'homme noir, esclave, garde une forme de noblesse car le non consentement de la femme violée lui permet de maintenir cette ultime forme d'existence, celle d'être un sujet désiré et désirant, celui qui occupe le véritable désir de la femme. J’ai pu ainsi montrer que la représentation de l'origine du métissage dans un viol permettait aux hommes de faire l'économie de la castration symbolique fondamentale que signifierait le métissage désiré par l'Africaine. Le viol donnerait en fait une version plus acceptable de l'origine du métissage, en permettant aux hommes de se construire dans un rôle de sujets désirés par les femmes violées, et aux femmes de se construire dans un rôle de femmes martyres et néanmoins mères fondatrices irréprochables des sociétés antillaises matrifocales.

Le rôle de la femme noire esclave est en effet présenté avec beaucoup d'ambivalence dans ces deux versions. Séduite, séductrice et traîtresse dans la première, elle devient victime et martyre dans la seconde. C'est d'ailleurs cette souffrance de femme violée qui conforte l'idée d'une femme résistante, capable de s'insurger contre l'oppresseur et de refuser d'intégrer les valeurs de la société coloniale. Le viol implique même que, fidèle à son compagnon africain d'infortune, elle puisse continuer de transmettre les valeurs et la culture du pays perdu. Au contraire, le métissage désiré fait de cette mère fondatrice, qui se satisfait des faveurs du Blanc et choisit cette rencontre comme stratégie de promotion sociale, une traîtresse qui sera ensuite la clé de voûte de l'intériorisation et l'incorporation des normes coloniales dans l'éducation des enfants; celle qui fera préférer le métissage et le blanchiment de la peau à la résistance africaniste ${ }^{25}$. Pourtant, la réalité historique a dû bien évidemment être plus complexe et mêler des situations et stratégies inextricables où la nécessité de survie impliquait des alliances indispensables, qui ne furent pourtant pas des prévarications. Comme le souligne Jacques André: « Il ne s'agit pas de ce que veut la mère mais de ce qu'elle ne peut pas ne pas vouloir. C'est toute la différence entre l'envie et le désir ${ }^{26}$.

\section{La méfiance généralisée}

L'idée de trahison et de fuite par rapport à une origine africaine ainsi dénigrée demeure associée à l'idée de métissage. Elle rejaillit sur les individus métis, autrefois appelés Mulâtres, dont la pensée populaire, présente encore dans des chansons et contes contemporains, considérait qu'ils se tourneraient toujours vers le haut de l'échelle 
sociale, en méprisant leurs origines africaines et noires. Déjà, au milieu du XIx ${ }^{e}$ siècle, "quelqu'un l'a dit avec vérité, écrivait Victor Schœlcher, un mulâtre hait son père et méprise sa mère $~_{27}$. Les alliances établies tout au long de l'histoire entre les libres de couleur, les Mulâtres et les Blancs pour l'exercice du pouvoir et pour se différencier des Nègres ont contribué à diviser la population de couleur et à reproduire des cascades de préjugés.

31 De nos jours, la méfiance est souvent de rigueur envers les individus dont la couleur de peau, la position sociale et l'origine peuvent faire craindre une attitude méprisante, dénigrante et infériorisante envers les Noirs : métis, négropolitains, mulâtres... Dans cette crainte du dédain potentiel, les métis sont le centre de toutes les suspicions. Définis aujourd'hui comme étant le produit de l'union entre un(e) Antillais(e) et un(e) Blanc (he), et de leurs oppositions biologiques et culturelles, ils rappellent par leur apparence et dans leur généalogie directe, proche et identifiable, immédiate et incontournable, le spectre de la relation au monde blanc, dominant, dépossédant, castrateur et prédateur. Les signes de l'apparence physique sont alors interprétés non comme le fruit d'un équilibre pacifique mais au contraire comme l'expression, à travers le corps, de la domination des caractéristiques blanches (le cheveu non plus crépu mais ondulé, les lèvres ou le nez plus fins, le parler pointu, la fréquentation des lieux de pouvoir...) et comme les indicateurs d'une appartenance sociale autre. Le groupe de référence et d'appartenance des métis n'étant pas clairement défini (est-ce celui de leur mère ou celui de leur père ?), l'on se méfie alors de ces personnages qui n'ont pas vraiment choisi leur camp et qui, instables sur cette corde raide, comme les Mulâtres leurs ancêtres, sont susceptibles de « renier leur race " à tout moment. De surcroît, les femmes métisses, dignes héritières des mulâ (traî) tresses d'antan, susceptibles de choisir des partenaires blancs dont elles connaissent déjà l'univers symbolique, replongent les hommes noirs dans l'angoisse d'une castration fondatrice réitérée.

\section{Vers une perméabilité des codifications de l'apparence}

Dans ce jeu de défiance, les chabins, nègres paradoxaux, montrent patte noire... à condition qu'ils prouvent qu'ils sont bien des Antillais à part entière, et qu'ils confirment par les actes le langage ventriloque de leur apparence corporelle. Une question permet de faire cette vérification: "vous êtes chabine ou métisse ? " adresse-t-on à celle dont on devine une origine blanche proche, dans son attitude (le fait de fumer, de faire du stop...), son apparence (des cheveux ondulés et non pas crépus), ou sa façon de parler (plus français que créole). Cette injonction à préciser son identité et son affiliation et à se positionner dans un camp ou dans l'autre témoigne de la nécessité d'identifier les personnes en fonction de leur origine.

Cependant, elle révèle aussi l'existence d'espaces d'incertitude et de négociations possibles, permettant à chacun de jouer avec des identifications multiples. Se présenter comme métis implique alors de garder une distance, une frontière et d'éviter une connivence, celle qui se partage directement avec les chabins. Se présenter comme chabin, c'est endosser une identité de membre d'une communauté dont on est censé connaître les rouages et les codifications, en y étant intégré, de recto. Mais il est possible de revendiquer l'une ou l'autre identité selon les circonstances, les interlocuteurs, les enjeux des interactions, en adaptant un comportement approprié et en maîtrisant les codifications correspondantes. Etre tour à tour chabin, métis, mulâtre, nègre et français 
relève de «la capacité à mêler ou à multiplier les masques et les appartenances " ${ }^{28}$. Ici, l'appartenance ne se résume plus à l'apparence physique mais tient compte aussi du comportement social et des interactions individuelles, qui opèrent comme un passeport identitaire. et d'appartenances et les capacités de négociations qu'elle laisse aux individus. Au-delà des injonctions sociales, les catégorisations sont en fait laissées à la discrétion des interactions individuelles et à la capacité des individus à franchir leurs limites. Cette perméabilité mesurée est parfois telle que les appellations « chabins » et « chabines » sont aujourd'hui facilement accordées par les jeunes générations à tout individu un tant soit peu clair de peau, ou de couleur de yeux, y compris aux métis, au grand dam des puristes qui s'insurgent contre de tels abus. Même des Européens blancs peuvent maintenant connaître cette désignation, estampillage non de leur généalogie, mais de leur transition identitaire, de leur aptitude à adopter les manières de vivre antillaises, sans les dénigrer. Cela semble être le signe d'un effacement progressif des frontières raciales, au profit d'un métissage social, même si celles-ci peuvent pourtant être vite rétablies en cas de conflit.

\section{Les négociations identitaires : une compétence métisse}

La capacité la plus attendue est donc la " compétence métisse ", c'est-à-dire la capacité à évoluer dans une dialectique permanente sur une échelle entre deux extrêmes, deux origines noire et blanche, figurant deux limites auxquelles il ne faut jamais adhérer complètement. Cette compétence métisse ${ }^{29}$, ou créole pourrais-je préciser, consiste alors à occuper subtilement les interstices de cet entre-deux, pour adopter plusieurs positions intermédiaires en fonction des enjeux des situations et en se défaisant des injonctions identitaires.

La compétence métisse correspond à cette capacité à jouer avec la couleur et sa signification, à contextualiser les apparences raciales pour s'adapter aux situations, à passer d'une norme à une autre. Cette capacité ne se déploie pas dans un espace social sans règles, sans contrainte, sans déterminants : précisément, elle repose sur la faculté de connaître et d'adapter ces codes. (...) Les acteurs manipulent et adhèrent à des codes qui leur permettent d'interpréter le comportement des autres et d'adopter pour eux-mêmes l'attitude la plus appropriée ${ }^{30}$.

Cette capacité à se positionner sur plusieurs registres, produite par la créolisation ellemême, évite l'enfermement dans des étiquetages coercitifs pour autant qu'elle ne remette pas en question la survie du groupe dans son ensemble. Si les apparences raciales apparaissent souvent comme les signes essentialistes non seulement d'une origine raciale mais aussi d'une appartenance sociale, elles sont néanmoins soumises aux pratiques subversives du métissage et de la créolisation, aux pratiques sociales et aux interactions individuelles qui les modèlent en retour.

Si les codifications raciales de l'apparence sont inscrites historiquement dans les corps des individus et dans l'univers symbolique des rapports sociaux, les dynamiques de créolisation obligent ces codifications à une certaine souplesse, dans laquelle chacun peut négocier en permanence l'identité qu'il choisit d'endosser, de revendiquer, en fonction des situations et des interlocuteurs. C'est pourquoi, malgré des assignations identitaires fortes et souvent contraignantes, le métissage et la créolisation suscitent chez l'individu une propension au passage, un goût de la transformation, une aptitude à la transition. Ce serait même le propre de la dynamique de créolisation de brouiller en permanence les 
apparences et les codifications et non de les figer, pour finir par les abolir... Ce fut en tout cas pour moi un exercice tout à fait singulier et formateur que d'être happée par la société guadeloupéenne à mon arrivée, en y étant perçue tantôt comme une chabine, une métisse, une mulâtresse, une saintoise, une albinos, une négropolitaine, une française... Ce fut une "vive expérience de l'identité $»^{31}$ qui n'a cessé d'alimenter mon travail de chercheure et ma façon de me positionner sur le terrain en développant moi aussi, autant que possible, cette compétence créole.

\section{BIBLIOGRAPHIE}

ANDRÉ Jacques, 1987, L'inceste focal dans la famille noire antillaise, Paris, PUF.

BERNAND Carmen, GRUZINSKI Serge, 1993, Histoire du nouveau monde. Les métissages, Paris, Fayard.

BONNIOL Jean-Luc, 1992, La couleur comme maléfice. Une illustration créole de la généalogie des Blancs et des Noirs, Paris, Albin Michel.

-, 2001, Les paradoxes du métissage, Paris, Editions du CTHS.

CHAMOISEAU Patrick, 1993, 1994, 2005, Une enfance créole I, II et III, Paris, Gallimard.

-, 2007, Un dimanche au cachot, Paris, Gallimard.

CONFIANT Raphaël, 1995, Ravines du devant jour, Paris, Gallimard.

2005, « Le mythe du chabin en Martinique, en Guadeloupe et en Haïti ». Communication au colloque de l'IRD, Regards croisés sur le métissage, Martinique.

COTTIAS Myriam, 1998, «L'oubli du passé'contre la 'citoyenneté' : troc et ressentiment à la Martinique (1848-1946) », in Fred CONSTANT, Justin DANIEL, (eds), Cinquante ans de départementalisation, Paris, L’Harmattan, p. 293-313.

CUNIN Elisabeth, 2001, « La compétence métisse. Chicago sous les tropiques ou les vertus heuristiques du métissage ", Sociétés Contemporaines, 42, p. 7-24.

DORLIN Elsa, 2006, La Matrice de la race. Généalogie sexuelle et coloniale de la Nation française, Paris, Editions La Découverte.

DORLIN Elsa et PARIS Myriam, 2006, « Genre, esclavage et racisme : la fabrication de la virilité », Contretemps, 16, p. 96-105.

FANON Franz, 1975 (1952), Peaux noires, masques blancs, Paris, Points-Seuil.

GLISSANT Edouard, 1999, « Métissage et créolisation », in Sylvie KANDÉ (dir.), Discours sur le métissage, identités métisses. En quête d'Ariel, Paris, L'Harmattan, p. 47-54.

GRACCHUS Fritz, 1980, Les lieux de la mère dans les sociétés afro-américaines, Paris, Editions Caribéennes, CARE.

LEIRIS Michel, 1955, Contacts de civilisations en Guadeloupe et en Martinique, Paris, Gallimard/Unesco. MOREAU DE SAINT-MÉRY, 1958 (1797), Description topographique, physique, civile, politique et historique de la partie française de l'Isle de Saint-Domingue, Paris, Larose. 
MULOT Stéphanie, 2007, « Le mythe du viol fondateur dans l'imaginaire antillais », Ethnologie française, XXXVII, 3, p. 517-524.

-, 2000, "Je suis la mère, je suis le père!» : l'énigme matrifocale. Relations familiales et rapports de sexes en Guadeloupe, Thèse de doctorat d'anthropologie sociale et ethnologie, dirigée par M. Godelier, EHESS, Paris.

PARIS Myriam, 2006, « La page blanche. Genre, esclavage et métissage dans la construction de la trame coloniale (La Réunion, XVIII ${ }^{\mathrm{e}}-\mathrm{XIX}{ }^{\mathrm{e}}$ siècle) », Cahiers du CEDREF, (Ré) articulation des rapports sociaux de sexe, classe et "race".

POURETTE Dolorès, 2002, « Le makòmè, masque de l'homosexualité masculine dans les mondes guadeloupéens », in R.-M. LAGRAVE (dir), Dissemblances. Jeux et enjeux du genre, Paris, L'Harmattan, p. 51-63.

-, 2006, Des Guadeloupéens en Ile de France. Identité, sexualité, santé, Paris, Karthala.

RÉGENT Frédéric, 2004, Esclavage, métissage, liberté. La Révolution française en Guadeloupe, Paris, Grasset.

SAADA Emmanuelle, 1995, « Le poids des mots, la routine des photos. Photographies de femmes martiniquaises : 1880-1930», Genèses, 21, p. 134-147.

-, 2007, Les Enfants de la colonie : les métis de l'Empire français entre sujétion et citoyenneté (1890-2000), Paris, La Découverte.

\section{NOTES}

1. Bonniol 1992 \& 2001 ; Régent 2004.

2. Dorlin 2006 ; Saada 2007.

3. Moreau de Saint-Méry (1952) s'en est fait le témoin pour Saint-Domingue, en répertoriant le nombre de parties noires et de parties blanches de chaque type.

4. Voir sur ce point les travaux de Myriam Cottias 1999.

5. Le terme « chabin » désigne initialement l'hybride né du croisement entre un mouton et une chèvre.

6. Le buste correspond à lestomak en créole, et désigne la cage thoracique, la poitrine, indépendamment des seins. "On chabin ni lestomak a Nèg» («Une chabine a un buste de Négresse »).

7. Leiris $1955: 161$.

8. Confiant 2005.

9. Surtout dans la littérature créole fondée sur la nostalgie antillaise. Raphaël Confiant (1995), parle aussi de son expérience personnelle de «mauvaise race de chabin-male-bougre». Voir aussi Patrick Chamoiseau, 1993, 1994, 2005 et 2007.

10. L'expression "tachetée comme une dinde» renvoie aux grains de peau en chapelet qui couvrent parfois les joues ou les pommettes des chabines, la comparaison imagée étant faite avec ces grains qui couvrent aussi la tête de la dinde.

11. Le terme "Kalasasa » est une onomatopée qui insiste sur le caractère rebelle et intempestif de ce type de chabine.

12. Chabines qui ont la particularité d'avoir une pilosité épaisse et drue, qui rappelle automatiquement dans cette représentation leur origine nègre.

13. On dit alors d'elles que « tout kô a yo chabin » (" elles sont chabines dans tout leur corps »). 
14. Je tiens cette dernière précision de mes entretiens de 1998 avec feu Guy Cornély, biologiste, botaniste et historien guadeloupéen.

15. Terme signifiant littéralement « ma commère ».

16. Sur l'homosexualité, voir Mulot 2000 et Pourette 2002 et 2006.

17. Dorlin et Paris 2006.

18. Saada $1995: 143$

19. Saada $1995: 143$.

20. Aucun autre terme désignant le type d'une personne n'est utilisé pour l'interpeler. Ce serait perçu comme dégradant.

21. Leiris $1955: 118$.

22. Le terme "métis", plus réducteur dans le vocable antillais qu'en français, sert aussi à désigner les enfants nés de l'union entre Blancs et Indiens. Il a remplacé dans le vocabulaire courant, le terme mulâtre, dans son acception initiale.

23. Glissant 1999.

24. Mulot 2007.

25. Fanon 1975 ; Gracchus 1980.

26. André $1987: 245$.

27. Leiris $1955: 151$

28. Bernand et Gruzinski $1993: 622$.

29. Cunin 2001.

30. Cunin 2001 : 17-18.

31. C'est ainsi que la qualifia Maurice Godelier, qui fut mon directeur de thèse, dans un échange personnel.

\section{RÉSUMÉS}

Aux Antilles françaises, les typologies du métissage mettent en exergue les personnages emblématiques que sont les chabins. L'analyse des représentations dont ils sont l'objet montre qu'ils symbolisent une alliance unique, précieuse et paradoxale au niveau de la race, du genre et du tempérament, entre les origines noire et blanche, alliance valorisée qui provoque la fierté populaire. À l'opposé, un métissage inquiétant, celui des "métis" rappelle les fondements traumatiques de la société coloniale et suscite une méfiance épidermique. Derrière des catégorisations et des assignations identitaires fortes émergent cependant des capacités de négociations identitaires qui permettent de sortir de tout enfermement radical.

In the French West Indies, the "chabins" symbolize a unique marriage between Blacks and Whites, whose cross-gender attitudes and behavior generate popular pride. On the contrary, the "metis" represents the more threatening figure of the crossbreed, who is viewed with automatic suspicion, since his existence evokes the traumatic origins of colonial society. Despite this identity labeling, creolisation creates a "metis" ability to negotiate, which prevents identity enclosure. 


\section{INDEX}

Index géographique : Antilles françaises

Keywords : chabins, French West Indies, identity labeling, métis ability, miscegenation, negotiation

Mots-clés : assignations identitaires, chabins, compétence métisse, métissage, négociation

\section{AUTEUR}

\section{STÉPHANIE MULOT}

Docteur en anthropologie de l'EHESS depuis 2000, maitresse de conférences en sociologie depuis 2005, chercheuse au LISST (UTM, CNRS, EHESS) université de Toulouse-Le Mirail, travaille depuis seize ans sur les sociétés antillaises. Ses premiers travaux ont porté sur les relations de parenté et la construction des identités sexuées dans les familles matrifocales guadeloupéennes. Elle a pu ainsi analyser les processus de construction identitaires, notamment dans les manifestations patrimoniales. Le métissage et la créolisation des savoirs, des pratiques et des identités étaient ainsi au cœur de sa réflexion. Elle travaille désormais sur la santé et la maladie aux Antilles, dans une démarche visant à tenir compte des différents niveaux d'analyse en montrant les conséquences des rapports de domination et des représentations de la santé sur les recours aux soins et les relations soignants/soignés. Les violences envers les femmes, la sexualité et surtout le sida sont ainsi ses objets d'études. 